\title{
Wave Attenuation by Sea Ice Turbulence
}

\author{
J. J. Voermans ${ }^{1}$, A. V. Babanin ${ }^{1}$, J. Thomson ${ }^{2}$, M. M. Smith ${ }^{2}$, and H. H. \\ Shen $^{3,4}$

\begin{abstract}
${ }^{1}$ Department of Infrastructure Engineering, University of Melbourne, Melbourne, Victoria, Australia ${ }^{2}$ Applied Physics Laboratory, Civil and Environmental Engineering, University of Washingon, Seattle, WA, USA

${ }^{3}$ Nanyang Environment and Water Research Institute, Nanyang Technological University, Singapore ${ }^{4}$ Department of Civil and Environmental Engineering, Clarkson University, Potsdam, NY, USA
\end{abstract}

\section{Key Points:}

- Turbulence may be an important dissipative mechanism of wave energy in the MIZ

- Turbulence-induced attenuation coefficient can be determined from measurements of wave energy and turbulence at a single location

- Turbulence dissipation is parameterized through characteristic wave and ice properties

This is the author manuscript accepted for publication and has undergone full peer review but has not been through the copyediting, typesetting, pagination and proofreading process, which may lead to differences between this version and the Version of Record. Please cite this article as doi: 10.1029/2019GL082945

Corresponding author: Joey Voermans, jvoermans@unimelb.edu.au 


\begin{abstract}
The dissipation of wave energy in the Marginal Ice Zone (MIZ) is often attributed to wave scattering and the dissipative mechanisms associated with the ice layer. In this study we present observations indicating that turbulence generated by the differential velocity between the sea ice cover and the orbital wave motion may be an important dissipative mechanism of wave energy. Through field measurements of under-ice turbulence dissipation rates in pancake and frazil ice, it is shown that turbulence induced wave attenuation coefficients are in agreement with observed wave attenuation in the MIZ. The results suggest that the turbulence-induced attenuation rates can be parameterized by the characteristic wave properties and a coefficient. The coefficient is determined by the ice layer properties.
\end{abstract}

\title{
1 Introduction
}

Ocean waves can penetrate hundreds of kilometers into vast sea ice covers before the ice fully attenuates their energy (Kohout, Williams, Dean, \& Meylan, 2014; Wadhams, Squire, Goodman, Cowan, \& Moore, 1988). Along the way, these waves impose stresses on the sea ice, enabling the waves to shape the region between the ice pack (referred to as the Marginal Ice Zone, MIZ) by breaking-up, moving and melting the ice (e.g. Squire, Dugan, Wadhams, Rottier, \& Liu, 1995). Ocean surface waves are therefore expected to accelerate Arctic ice retreat in the near future due to seasonal opening of the Arctic seas (Q. Liu, Babanin, Zieger, Young, \& Guan, 2016; Thomson \& Rogers, 2014) and, with loss of the seasonal MIZ around Antarctica, ocean swell may contribute to the disintegration of the Antarctic ice shelves (Massom et al., 2018). To include these effects in weather and climate forecasting models, spectral wave models require processbased parameterizations of wave attenuation to predict the wave field transformation in the MIZ. The physical processes that dictate the dissipation of wave energy by sea ice are, however, still under debate.

Various processes are currently identified as contributors to the observed wave attenuation in the MIZ and are parameterized in spectral models, including wave scattering (e.g Montiel, Squire, \& Bennetts, 2016; Wadhams et al., 1988); ice layer interactions, where the ice is often parameterized as a viscoelastic layer (Mosig, Montiel, \& Squire, 2015; Wang \& Shen, 2010); and under-ice turbulence (e.g. A. Liu \& Mollo-Christensen, 1988). The first is not a dissipative process as the reflection of waves by solitary ice floes merely alters the direction of wave energy, such that part of the energy is scattered back into the open ocean while the rest is transmitted further into the MIZ. Complex dissipation processes associated with the ice layer are typically parameterized through viscoelastic theories, where dissipation can be regulated through the mechanical properties of the ice layer model. Models for wave attenuation based on parameterization of wave scattering or dissipation by representing the sea ice cover as a viscoelastic layer have been able to explain, in part, wave observations in the MIZ. However, as they often require careful calibration of the modeled ice layer characteristics, the properties of which vary greatly in both space and time, our predictive abilities are severely restricted without advancement in the physical description of processes that actually drive wave attenuation in the MIZ. Additionally, recent experimental observations in the laboratory, where measured material properties of the ice layer were used as input for the viscoelastic model, revealed large discrepancies between the measured wave attenuation and those predicted by parameterizing the ice cover as a viscoelastic layer (Sree, Law, \& Shen, 2018).

Under-ice turbulence has remained a relatively unexplored dissipative mechanism since the study of A. Liu and Mollo-Christensen (1988) (with some exceptions, such as Ardhuin, Sutherland, Doble, and Wadhams (2016); Kohout, Meylan, and Plew (2011); Shen and Squire (1998)). Waves receive energy by wind, while they lose energy through the production of turbulence. Turbulence is generated through boundary layer develop- 
ment under the ice, wake formation within the ice-layer around solitary ice floes (or form drag, e.g. Kohout et al., 2011) and ice-floe collisions (Rabault, Sutherland, Jensen, Christensen, \& Marchenko, 2019). Through order of magnitude estimates (A. Liu \& MolloChristensen, 1988) and spectral model calibration (Ardhuin et al., 2016), the generation of under-ice turbulence shows reasonable agreement with in-situ wave attenuation observations, or is shown to be much larger than estimates of energy dissipation by other attenuation processes, including scattering (Shen \& Squire, 1998). The few turbulence estimates presently available are, however, not substantiated by any turbulence measurements in the field as measuring turbulent properties in these extreme environments is a major challenge. Hence, it remains uncertain whether turbulence is an important contributor to the attenuation of wave energy and should be included in spectral wave models.

The objective of this study is to assess the importance of under-ice turbulence in attenuating wave energy in the MIZ. Here, in-situ measurements of the turbulence dissipation rates from the Arctic Sea State Program are used to estimate turbulence-induced wave attenuation under mixtures of pancake and frazil ice and are compared against measurements of wave attenuation.

\section{Turbulence-Induced Wave Attenuation Coefficient}

Wave energy in the MIZ is known to decrease exponentially with distance into the ice cover (Wadhams et al., 1988):

$$
S(f, x+\Delta x)=S(f, x) \exp (-\alpha \Delta x)
$$

where $x+\Delta x(\mathrm{~m})$ is the distance in the direction of wave propagation relative to an arbitrary point $x$ within the MIZ, $S(f, x)$ is the spectral energy density at the position $x$ and $\alpha(1 / \mathrm{m})$ is the frequency dependent wave attenuation coefficient. When the vertical structure of the turbulent kinetic energy (TKE) dissipation rates $\varepsilon$ is known, the total dissipation of TKE $D\left(\mathrm{~W} / \mathrm{m}^{2}\right)$ is determined by:

$$
D=\int_{-\delta}^{0} \rho \varepsilon d z
$$

where $\varepsilon$ is the TKE dissipation rate of turbulence generated by wave-ice interactions, $\delta$ is the thickness of the turbulent boundary layer, $\rho$ is the density of the ocean water and $z$ is the distance from the sea ice interface. If the wave attenuation is dominated by the turbulent shear stress between the sea ice interface and the orbital motion of the fluid, the change of wave energy in the direction of wave propagation is:

$$
E(x+d x)-E(x)=-D d t=-D d x / c_{g}
$$

where $c_{g}$ is the group velocity of a characteristic wave period, $E(x)=\rho g \int S(f, x) d f$ $\left(\mathrm{Ws} / \mathrm{m}^{2}\right)$ is the total wave energy at a distance $x$. Note that as a first order approximation, wind input is ignored as source of wave energy in Eq. 3. Total wave energy (instead of the spectral energy) is considered here as the wave frequency dependence of $\varepsilon$ cannot be determined from the turbulence measurements by itself, as all turbulence generated below the sea ice interface is subjected to the turbulence energy cascade.

Under the assumption that most of the measured TKE dissipation rate originates from a narrow range of frequencies, Eqs 1 and 3 can be combined to yield:

$$
-D / c_{g}=-\alpha_{t} E(x) \exp \left(-\alpha_{t} \Delta x\right)
$$

where the subscript ' $t$ ' in $\alpha_{t}$ refers to the turbulence-induced attenuation of wave energy. As $\alpha_{t}$ corresponds to the attenuation of the total wave energy, $\alpha_{t}$ is here simply a function of the characteristic frequency of the spectral energy density $S(f, x)$, for example, 
the mean or peak frequency. If both turbulence and waves are then measured at the same location (i.e. $\Delta x=0$ ), it follows from Eq. 4 that the turbulence-induced wave attenuation coefficient can be determined by:

$$
\alpha_{t}=\frac{D}{c_{g} E(x)}
$$

It follows from Eq. 5, that concurrent measurements of turbulence and waves are required to determine the turbulence-induced attenuation coefficient. It should be stressed that $\varepsilon$ (and consequently $\alpha_{t}$ ) is critically defined here as the TKE dissipation rate of turbulence generated through wave-ice interactions only.

Hereafter, the mean wave period $T_{m 01}=m_{0} / m_{1}$ (where $m_{0}$ and $m_{1}$ are the zeroth and first-order moment of the energy spectrum) is the characteristic wave period used to determine $c_{g}$. As the open water dispersion relation is likely to hold for waves in the MIZ with periods in the range of 3-10 s (Collins, Doble, Lund, \& Smith, 2018), a linear paradigm is used to model the wave propagation speed.

\section{Methods}

The data used in this study were obtained during the Arctic Sea State Program in the Beaufort and Chukchi Seas. The measurement campaign included arrays of wave buoys deployed in frazil and/or pancake ice during 7 Wave Experiments (WE) from October to November 2015. A summary of the Sea State Program and measurement campaign can be found in Thomson (2015) and Thomson et al. (2018). Here, we focus on a brief description of the SWIFT drifters and the processing of data.

SWIFT is a wave following drifting buoy which simultaneously measures the ocean surface motion and the under-ice turbulent velocity components. Part of the drifters were mounted with an acoustic Doppler current profiler (ADCP, Nortek Aquadopp HR) in upward direction to measure profiles of the turbulent velocity fluctuations within $1 \mathrm{~m}$ from the surface. After quality control measures, including ice-masking, the measured turbulent velocity components of the drifters were used to estimate profiles of the turbulence dissipation rate through a second-order structure function, as per Smith and Thomson (2019). This method has been validated against independent dissipation rate measurements from a second instrument (single-point acoustic Doppler velocimeter, ADV) mounted on a SWIFT drifter, where dissipation rates were estimated by fitting Kolmogorov's $-5 / 3$ law to the inertial subrange of the measured velocity spectrum (Thomson, 2012). The reader is referred to Smith and Thomson (2019) for a detailed description on the processing of the data obtained by the SWIFT buoys.

The SWIFT buoys equipped with upward looking ADCP's were deployed in tandem with SWIFT buoys with a downward looking ADCP, measuring velocity profiles from 1.5 to $21 \mathrm{~m}$ below the ocean surface. By collocating the upward and downward looking ADCP's, the mean relative velocity between the ice and the ocean $\Delta U$ is taken as the velocity in the upper bin of the downward looking ADCP (Smith \& Thomson, 2019) and will be used to exclude mean shear between the ice and the upper ocean (drift) as a significant source of turbulence.

As the SWIFT buoys were not continuously drifting in the ice covers, only parts of the wave experiments are considered here. Based on the images captured by the camera mounted on the SWIFT buoys, the buoys were within the ice covers on: WE3, 1013 Oct. (SWIFT 9, 11, 14 and 15); WE4, 17-18 Oct. (SWIFT 11, 14 and 15); WE6, 2324 Oct. (SWIFT 9, 11 and 12); and WE7, 31 Oct-1 Nov (SWIFT 9, 11, 13 and 15). A summary of the wave and ice conditions during these deployments is provided in Table S1. 


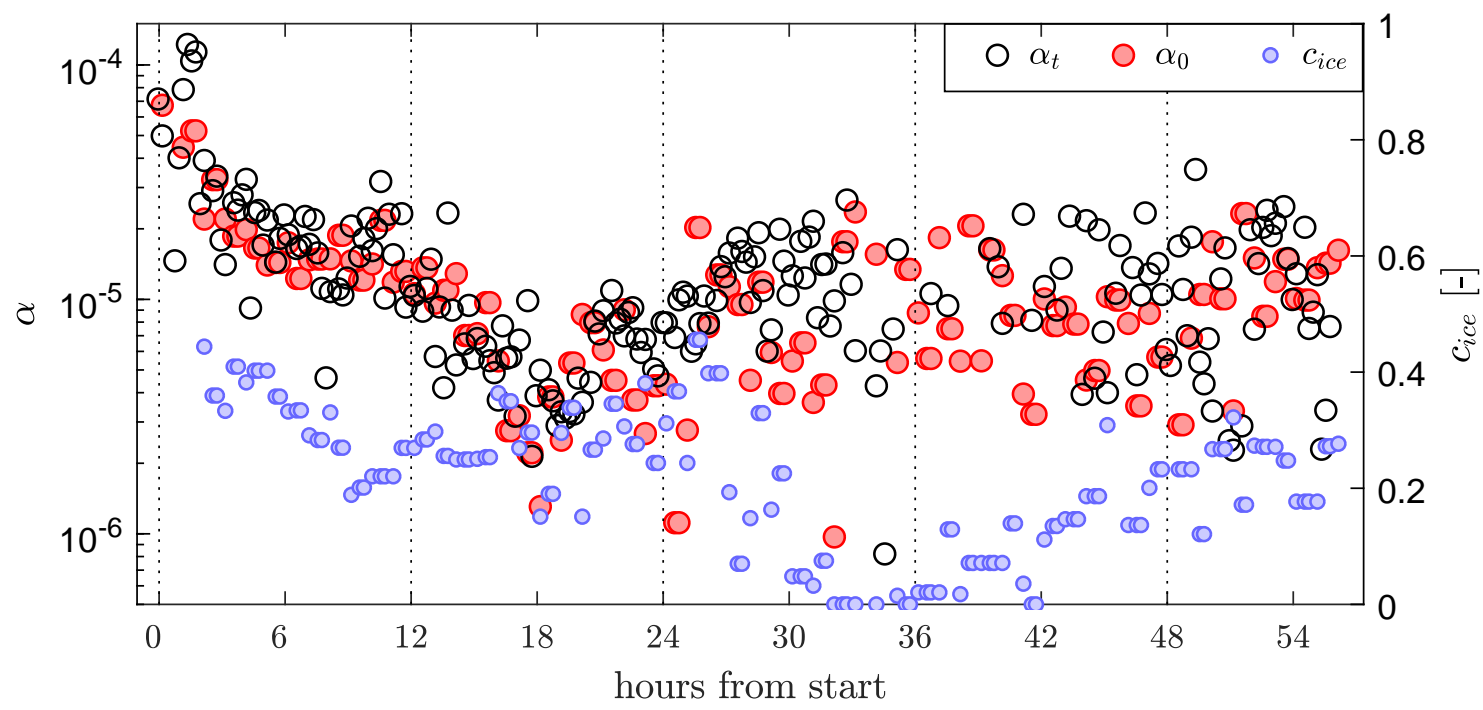

Figure 1. Time series of the turbulence-induced wave attenuation $\alpha_{t}$ (black), the observed total wave attenuation $\alpha_{0}$ (red) and ice concentration $c_{i c e}$ (blue) during the deployment of SWIFT 14, WE3, on 11-13 October. Note that $c_{i c e}$ (obtained from AMSR2) does not always agree to visual observations of ice concentrations by camera images.

By deploying multiple SWIFT buoys at different positions within the MIZ, the total wave attenuation $\alpha_{0}$ is determined by evaluating the total wave energy decay between buoy pairs:

$$
\alpha_{0}=\frac{1}{\Delta x} \ln \left(\frac{\int S(f, x) d f}{\int S(f, x+\Delta x) d f}\right)
$$

where $\Delta x=\Delta x_{\theta} \cos \left(\theta_{m}-\theta\right)$ is the distance between the buoypair along the mean direction of the wave field, $\Delta x_{\theta}$ is the great-circle distance between the buoys, $\theta_{m}$ is the mean wave direction and $\theta$ is the bearing angle of the buoy pair. Quality control criteria for $\alpha_{0}$ from Cheng et al. (2017) were adopted, though in this study the maximum allowable angle between $\theta_{m}$ and $\theta$ is set to $70^{\circ}$. Additionally, a minimum buoy pair distance of $\Delta x_{\theta}=250 \mathrm{~m}$ is enforced to ensure energy dissipation across the full spectrum can be reasonably measured. Only buoy pairs containing two SWIFT drifters and at least one SWIFT drifter with a upward looking ADCP were used to determine $\alpha_{0}$. When multiple observations of $\alpha_{0}$ were available at one instance, $\alpha_{0}$ is taken as the mean of the logarithms of these observations, as equally valid measurements can be more than one order of magnitude apart. Note that, similarly as in Eq. 3, we neglect wind input as possible source of wave energy in Eq. 6. The ice concentration $c_{i c e}$ during the wave experiments are approximated using AMSR2 (Spreen, Kaleschke, \& Heygster, 2008).

\section{Results and Discussion}

\subsection{Example time series of $\alpha_{t}$ and $\alpha_{0}$}

Figure 1 shows a comparison of observed total wave attenuation $\alpha_{0}$ and estimated turbulence-induced wave attenuation $\alpha_{t}$ for a deployment during WE3 on 11 October. Close agreement between $\alpha_{t}$ and $\alpha_{0}$ is observed up to $t=24$ hours from the start $\left(r^{2}=0.80\right.$, in contrast, $r^{2}=0.54$ for the full time series). Beyond $t=24 \mathrm{~h}$, both $\alpha_{t}$ and $\alpha_{0}$ vary considerably in time but remain, nevertheless, similar in order of magnitude. Although correlation between $\alpha_{t}$ and $c_{i c e}$ is weak for the time series, comparable trends can be ob- 


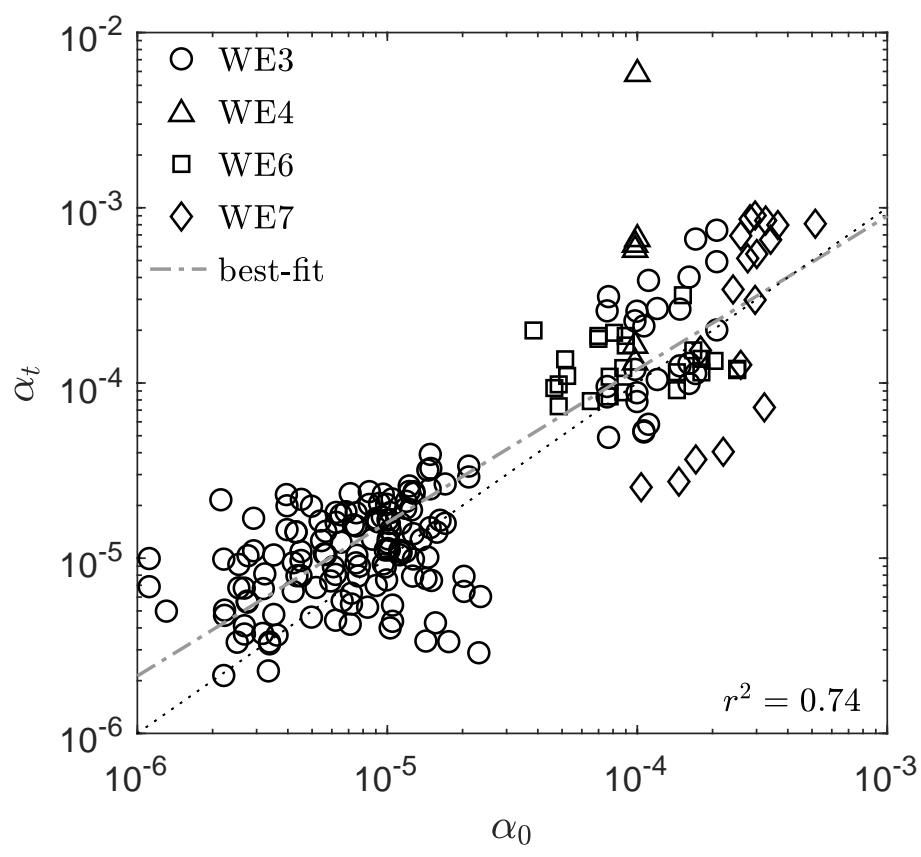

Figure 2. Comparison of the turbulence-induced wave attenuation $\alpha_{t}$ (Eq. 5) against the total wave attenuation $\alpha_{0}$ (based on the mean of buoy-pair observations). Markers identify the different wave experiments. Best-fit to the data is given by the dash-dotted line.

served during the first 12 hours, where an initial decrease in wave attenuation corresponds to a decrease in ice concentration, after which both $\alpha_{t}$ and $c_{i c e}$ seem to increase till around 24 hours from the start. Estimates of ice concentrations after $t=24 \mathrm{~h}$ do not follow trends of $\alpha_{t}$, however, such estimates should be interpreted with caution, as ice cover heterogeneity can occur at scales smaller than the resolution of AMSR2. For instance, while no ice is present around $t=36 \mathrm{~h}$ according to AMSR2 approximations (see also Figure S1), images captured by the SWIFT buoy suggest a mixture of frazil and pancake ice to be present from $t=29 \mathrm{~h}$ till $t=40 \mathrm{~h}$ (see Figure S2 for images captured during the deployment). Although daylight limits observations of conditions throughout the entire deployment, based on daytime images, it is hypothesized that ice conditions at the air-ice interface remain relatively constant after $t=24 \mathrm{~h}$. This would be consistent with the trend of $\alpha_{t}$ during this time. The correlation between $\alpha_{t}$ and $\alpha_{0}$ seen for this deployment demonstrates that turbulence generated through wave-ice interactions can explain wave energy dissipation in the MIZ.

\subsection{Overall comparison of $\alpha_{t}$ and $\alpha_{0}$}

The total wave attenuation is then compared against the turbulence-induced wave attenuation for all wave experiments over half-hour periods (Figure 4). Good agreement is observed across the wave experiments and over a wide range of wave attenuation coefficients $\left(r^{2}=0.74\right)$. The results include a correlation associated with scaling both axes by wave energy; however, this correlation only explains $19 \%$ of the variance of the signal. Thus, it suggest that turbulence-induced wave attenuation may be an important dissipation mechanism of wave energy in the MIZ.

Central in this study is the assumption that the dominant source of measured turbulence under the ice is from wave-ice interactions. A few physical processes could be 
responsible for the production of TKE below the ice layer, including turbulence generation by ice floe collisions (which can lead to jet-like injections of fluid into the ice layer), overwash, wake flow around ice formations, keel ridges, TKE production by the mean shear (drift) at the sea ice interface and by wind related processes. Note that wind can only impact the production of turbulence indirectly through drift currents and wind generated waves. As the TKE dissipation rate $D$ scales with the cube of the characteristic velocity scale of the dominant physical process, turbulence input through wind-induced wave generation are expected to scale with the wind speed $D \propto U_{10}^{3}$, while turbulence production by the mean shear (including wind-induced drift) scales with the differential velocity between the ice and the upper ocean, i.e. $D \propto \Delta U^{3}$. For the current field experiments, there is no correlation between $D$ and $\Delta U\left(r^{2}=0.05\right)$, and limited correlation between $D$ and wind speed $\left(r^{2}=0.32\right)$. Note that this correlation could be spurious, as the wind field is intrinsically linked to the waves and, as a result, correlated to wave-ice interactions as well, implying that wave-ice interactions dominate turbulence production in this study. This is consistent with the strong correlation observed between $\alpha_{0}$ and $\alpha_{t}$ (i.e. Figures 1 and 4), as only turbulence generated through wave-ice interaction processes can contribute to wave attenuation in the MIZ. Hence, turbulence induced wave attenuation could be an important dissipative process of wave energy in the MIZ.

Note that observations of $\alpha_{t}$ can exceed the measured wave attenuation (i.e. by up to a factor of two for very low wave attenuation rates, see best-fit to the data in Figure 4 ), which implies that other processes and/or energy sources are present. Due to the lack of correlation between $D$ and $\Delta U$, the most likely source of wave energy or turbulence is by local wind input (Smith \& Thomson, 2019; Zippel \& Thomson, 2016), a source term that is neglected in this study to a first order approximation in both $\alpha_{t}$ and $\alpha_{0}$ (i.e. Eqs. 3 and 6). In particular, Li et al. (2017) determined that wave energy input through wind in the MIZ should be considered and observed that the rate of energy transfer is dependent on the strength of the wind field.

\subsection{Modeling turbulence dissipation under sea ice}

To estimate the dissipation of turbulence within the wave boundary layer (WBL), the presence of a balance between TKE production $(P)$ and TKE dissipation is adopted (e.g. Tennekes \& Lumley, 1972):

$$
\varepsilon \approx P \approx-\overline{u^{\prime} w^{\prime}} \frac{d \bar{u}}{d z}
$$

where $\overline{u^{\prime} w^{\prime}}$ is the ensemble averaged Reynolds stress and $d \bar{u} / d z$ the vertical mean velocity gradient. The Reynolds stress is approximated by $-\overline{u^{\prime} w^{\prime}} \approx u_{*}^{2}$ with $u_{*}$ being the shear velocity, while the velocity profile in the WBL is simplified as linear such that $d \bar{u} / d z \approx$ $u_{\text {orb }} / \delta$, where $u_{o r b}=\pi H_{m 0} / T_{m 01}$ is the representative wave orbital velocity and $\delta$ is the thickness of the WBL. By substitution of the preceding into Eq. 7 and integration of the turbulence dissipation rate over the WBL thickness, the dissipation rate of TKE per square meter surface area can then be estimated by:

$$
D_{W B L} \approx b_{1} \int_{-\delta}^{0}-\rho u_{*}^{2} \frac{u_{\text {orb }}}{\delta} d z=\rho u_{*}^{2} u_{o r b}
$$

where $b_{1}$ is a constant of proportionality. Using the analogy between a sea ice interface and a wave bottom boundary layer, the wave friction velocity is considered to be proportional to the wave orbital velocity (e.g. Madsen, Poon, \& Graber, 1989). Thus, the dissipation of TKE (Eq. 8) can be interpreted as the product of the interfacial stress $\left(u_{*}^{2}=\right.$ $C_{D} u_{\text {orb }}^{2}$ ) and the characteristic velocity scale $u_{\text {orb }}$ (e.g. Smith \& Thomson, 2019). The generation of turbulence by the differential velocity of the orbital wave motion and the ice layer is then proportional to $u_{o r b}^{3}$. Combining the drag coefficient $C_{D}$ and constant 

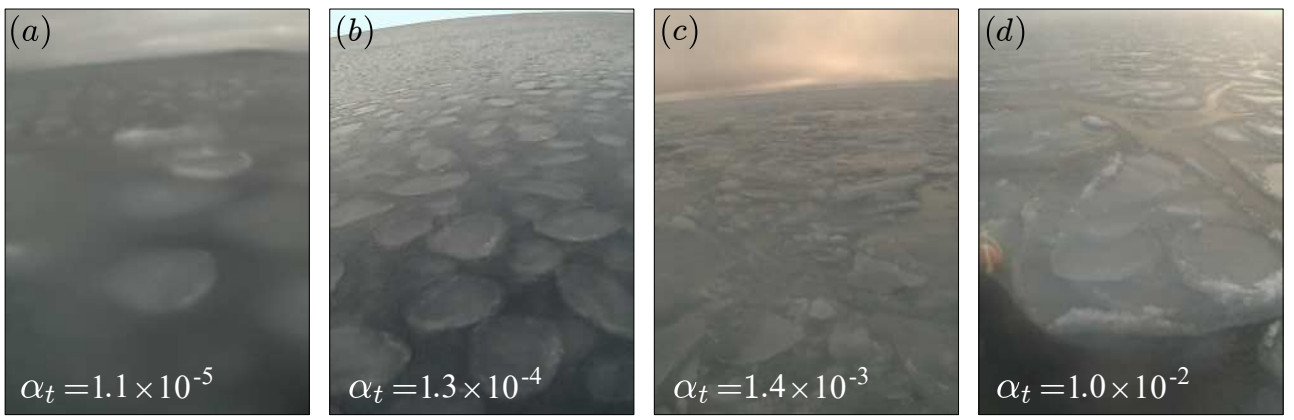

Figure 3. Images taken by the SWIFT drifters during deployment for varying ice conditions and measured turbulence induced attenuation rates: (a) WE3 SWIFT 14; (b) WE3 SWIFT 11; (c) WE6 SWIFT 12; (d) WE4 SWIFT 11.

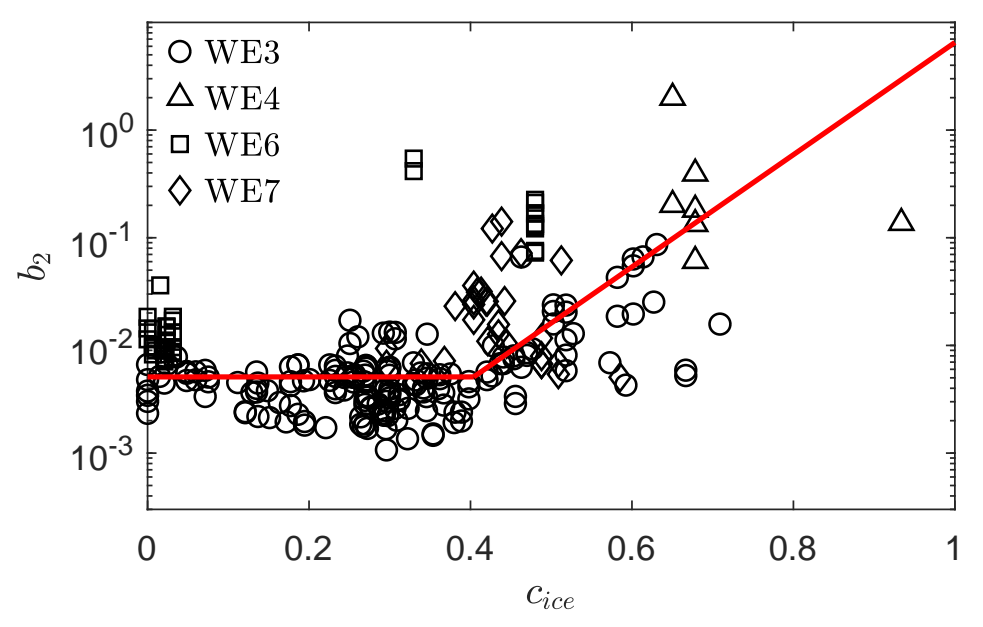

Figure 4. Variation of coefficient $b_{2}$ (see Eq. 9) with ice concentration $c_{i c e}$ for all wave experiments. For $c_{i c e}<0.4, b_{2}=3.6 \times 10^{-4}$, and for $c_{i c e} \geq 0.4, b_{2}=1.0 \times 10^{-7} \exp \left(20 c_{i c e}\right)$.

$b_{1}$ into a new coefficient $b_{2}, b_{2}$ can be defined as:

$$
D_{W B L}=\rho b_{2} u_{o r b}^{3}=\rho b_{2}\left(\frac{\pi H_{m 0}}{T_{m 01}}\right)^{3}
$$

The coefficient $b_{2}$ can be interpreted as the ratio of TKE dissipation rate to the kinetic energy of the local wave state and, hence, represents the relative dissipation rate of TKE.

While the orbital velocity is a reasonable approximation of the velocity scale that characterizes under-ice turbulence, the fundamental velocity scale that defines $D_{W B L}$ is the differential velocity between the ice and the wave orbital velocity. Any deviation of the orbital wave motion by wave-following sea ice (for instance, as is often the case with pancake ice) is therefore embedded in the coefficient $b_{2}$. Thus, $b_{2}$ is not only expected to be a function of ice roughness, but also of wave and other ice properties. In the case of loose ice, this includes the draft of the ice floes (or ice thickness), their diameter and the ice type. In particular, Rogers et al. (2016) found that wave dissipation rate $\alpha_{0}$ sorted well by ice type. Similarly, we observed a higher turbulence-induced attenuation rate $\alpha_{t}$ in more consolidated pancake ice (Figure 3). As ice concentration is the most easily measurable characteristic of sea ice, the variation of $b_{2}$ is compared against the ice concentration in Figure 4. The results suggest that for $c_{i c e}<0.4$ the dissipation rates of TKE 
become nearly independent of $c_{i c e}$. Above $c_{i c e}=0.4$, however, dissipation rates tend to increase with ice concentration. Estimates of the coefficient $b_{2}$ in this study correspond well to equivalent coefficients observed by others. For instance, Lu, Li, Cheng, and Leppäranta (2011) report ice-ocean drag coefficients ranging from $1 \times 10^{-4}$ to $5 \times 10^{-2}$ for a broad variety of sea ice conditions. Additionally, Gallaher et al. (2016) found ice drag coefficients in the range of $3 \times 10^{-3}$ to $6 \times 10^{-3}$ for first-year ice in the Arctic MIZ.

While ice concentration might be a good representative measure for the dominant roughness length scale for small ice concentrations, other roughness scales are likely to dominate friction when the sea ice cover turns more solid. Thus, the scatter in $b_{2}$ in Figure 4 is likely a result of variation in ice type and thickness. It should be noted that the ice concentrations obtained by AMSR2 are spatially averaged estimates of the local ice conditions. In particular, due to the resolution of AMSR2, local ice conditions can deviate significantly from those obtained of AMSR2. For instance, based on the estimates of AMSR2 for the two outliers during WE6 (see two squares in Figure 4), the ice concentration was estimated to be 0.33 , while images captured at these instant suggest considerably higher ice concentrations (see corresponding ice cover in Figure 3c).

Although the results suggest that turbulence induced by under-ice friction becomes relevant for ice concentration above 0.4 only, production of turbulence for lower ice concentration cannot necessarily be ignored. Specifically, the observed value of $b_{2} \approx 4 \times$ $10^{-4}$ corresponds well to the magnitude of turbulence in open water swell seas, i.e. $b_{2} \approx$ $7 \times 10^{-4}$ (Babanin, 2012). As $b_{2}$ represents the relative dissipation rate of TKE (i.e. see Eq. 9), the relative dissipation rate of TKE under sea ice covers for $c_{i c e}>0.4$ can be larger than that observed in open water. This also substantiates that there are no other significant sources of turbulence in this study, as they would have led to $b_{2}$ being larger than that observed for swell.

The results of this study, therefore, imply that turbulence generated by the differential velocity between the orbital wave motion and the ice layer may be an important dissipative mechanism for wave energy in the MIZ. Thus, turbulence should be added to the list of wave-ice interaction processes in spectral wave models. A simple relation is suggested here where the dissipation rate of TKE per square meter surface area can be estimated using Eq. 9, by the following model for $b_{2}$ :

$$
\begin{aligned}
& b_{2}=3.6 \times 10^{-4} \quad \text { for } \quad c_{i c e}<0.4 \\
& b_{2}=1.0 \times 10^{-7} \exp \left(20 c_{i c e}\right) \quad \text { for } \quad c_{\text {ice }} \geq 0.4
\end{aligned}
$$

However, implementation of the above model into spectral wave models is complicated by the integral approach that was inevitably adopted to measure and determine the TKE dissipation rate, rather than a spectral solution to $D$, i.e. varying with wave frequency.

While the TKE dissipation rate in this study is parameterized using wave and ice properties, alternative parameterizations based on wind speed can reproduce the observed turbulence as well (e.g. Smith \& Thomson, 2019). This is not surprising as wind and waves are inevitably correlated and is particularly true when processes are averaged over large spatial scales or are observed at the edge of the MIZ. Additional studies are therefore required to further elucidate the complex interaction between wind, waves and the ice and connect these bottom-up (in terms of the waves) and top-down (in terms of the wind) approaches to a unified framework of momentum and energy conservation across the airice-sea interface. An experimental laboratory study in the absence or presence of wind may progress our understanding of the interactions across the ice layer, including the scaling of wind-input, ice drift and the impact of ice-layer properties on the coefficient $b_{2}$. Further efforts are required to define the wave-induced attenuation coefficient $\alpha_{t}$ as a function of wave frequency, as it is well known that wave attenuation decreases with increasing wave period (e.g. Cheng et al., 2017; Meylan et al., 2018; Rogers et al., 2016; Wadhams et al., 1988). 


\section{Conclusion}

Through field measurements of turbulence dissipation rates under pancake and frazil ice covers, we show that turbulence generated by the differential velocity between the ice and the orbital wave motion may explain the observed attenuation of wave energy in the MIZ. The large variability of the attenuation rates is argued to be the result of temporal and spatially varying ice conditions. Our results suggest that turbulence-induced wave attenuation rates can be parameterized through characteristic wave properties and a coefficient $\left(b_{2}\right)$ where $b_{2}$ remains constant for ice concentrations $c_{i c e}$ below $0.4\left(b_{2}=\right.$ $\left.3.6 \times 10^{-4}\right)$ and increases for $c_{i c e}>0.4$ as $b_{2}=1.0 \times 10^{-7} \exp \left(20 c_{i c e}\right)$. More experiments are, however, required to quantify the coefficient $b_{2}$ in terms of ice layer properties and determine the dependence of the turbulence-induced wave attenuation coefficient on wave frequency.

\section{Acknowledgments}

This research was supported by the DISI Australia- China Centre through Grant ACSRF48199. AVB acknowledges support from the US Office of Naval Research grant N0001417-1-3021, and HHS acknowledges support from the US Office of Naval Research through grant N00014-17-1-2862. Data used in this study was colleced with the support of Office of Naval Research award N00014-13-1-0284, as part of the "Sea State and Boundary Layer Physics of the Emerging Arctic Ocean" Departmental Research Initiative. Data is available at www.apl.uw.edu/arcticseastate and hdl.handle.net/1773/41864. We would like to thank the anonymous reviewers for their comments and suggestions.

\section{References}

Ardhuin, F., Sutherland, P., Doble, M., \& Wadhams, P. (2016). Ocean waves across the arctic: Attenuation due to dissipation dominates over scattering for periods longer than 19 s. Geophysical Research Letters, 43(11), 5775-5783. doi: 10.1002/2016GL068204

Babanin, A. V. (2012). Swell attenuation due to wave-induced turbulence. In Asme 2012 31st international conference on ocean, offshore and arctic engineering (pp. 439-443).

Cheng, S., Rogers, W. E., Thomson, J., Smith, M., Doble, M. J., Wadhams, P., ... Shen, H. H. (2017). Calibrating a viscoelastic sea ice model for wave propagation in the arctic fall marginal ice zone. Journal of Geophysical Research: Oceans, 122(11), 8770-8793. doi: 10.1002/2017JC013275

Collins, C., Doble, M., Lund, B., \& Smith, M. (2018). Observations of surface wave dispersion in the marginal ice zone. Journal of Geophysical Research: Oceans, 123(5), 336-3354. doi: 10.1029/2018JC013788

Gallaher, S. G., Stanton, T. P., Shaw, W. J., Cole, S. T., Toole, J. M., Wilkinson, J. P., ... Hwang, B. (2016). Evolution of a canada basin ice-ocean boundary layer and mixed layer across a developing thermodynamically forced marginal ice zone. Journal of Geophysical Research: Oceans, 121(8), 6223-6250. doi: 10.1002/2016JC011778

Kohout, A. L., Meylan, M. H., \& Plew, D. R. (2011). Wave attenuation in a marginal ice zone due to the bottom roughness of ice floes. Annals of Glaciology, 52(57), 118-122. doi: 10.3189/172756411795931525

Kohout, A. L., Williams, M. J. M., Dean, S. M., \& Meylan, M. H. (2014). Storminduced sea-ice breakup and the implications for ice extent. Nature, 509, 604607. doi: $10.1038 /$ nature 13262

Li, J., Kohout, A. L., Doble, M. J., Wadhams, P., Guan, C., \& Shen, H. H. (2017). Rollover of apparent wave attenuation in ice covered seas. Journal of Geophysical Research: Oceans, 122(11), 8557-8566. doi: 10.1002/2017JC012978

Liu, A., \& Mollo-Christensen, E. (1988). Wave propagation in a solid ice pack. 
Journal of physical oceanography, 18(11), 1702-1712. doi: doi.org/10.1175/ 1520-0485(1988)018<1702:WPIASI $\rangle 2.0 . C O ; 2$

Liu, Q., Babanin, A. V., Zieger, S., Young, I. R., \& Guan, C. (2016). Wind and wave climate in the arctic ocean as observed by altimeters. Journal of Climate, 29(22), 7957-7975. doi: 10.1175/JCLI-D-16-0219.1

Lu, P., Li, Z., Cheng, B., \& Leppäranta, M. (2011). A parameterization of the iceocean drag coefficient. Journal of Geophysical Research: Oceans, 116(C7). doi: 10.1029/2010JC006878

Madsen, O. S., Poon, Y.-K., \& Graber, H. C. (1989). Spectral wave attenuation by bottom friction: Theory. In Coastal engineering 1988. doi: 10.1061/9780872626874.035

Massom, R. A., Scambos, T. A., Bennetts, L. G., Reid, P., Squire, V. A., \& Stammerjohn, S. E. (2018). Antarctic ice shelf disintegration triggered by sea ice loss and ocean swell. Nature, 383-389. doi: 10.1038/s41586-018-0212-1

Meylan, M. H., Bennetts, L., Mosig, J., Rogers, W., Doble, M., \& Peter, M. A. (2018). Dispersion relations, power laws, and energy loss for waves in the marginal ice zone. Journal of Geophysical Research: Oceans, 123(5), 33223335. doi: 10.1002/2018JC013776

Montiel, F., Squire, V. A., \& Bennetts, L. G. (2016). Attenuation and directional spreading of ocean wave spectra in the marginal ice zone. Journal of Fluid Mechanics, 790, 492-522. doi: 10.1017/jfm.2016.21

Mosig, J. E. M., Montiel, F., \& Squire, V. A. (2015). Comparison of viscoelastictype models for ocean wave attenuation in ice-covered seas. Journal of Geophysical Research: Oceans, 120(9), 6072-6090. doi: 10.1002/2015JC010881

Rabault, J., Sutherland, G., Jensen, A., Christensen, K. H., \& Marchenko, A. (2019). Experiments on wave propagation in grease ice: combined wave gauges and particle image velocimetry measurements. Journal of Fluid Mechanics, 864, 876-898. doi: 10.1017/jfm.2019.16

Rogers, W. E., Thomson, J., Shen, H. H., Doble, M. J., Wadhams, P., \& Cheng, S. (2016). Dissipation of wind waves by pancake and frazil ice in the autumn beaufort sea. Journal of Geophysical Research: Oceans, 121(11), 7991-8007. doi: 10.1002/2016JC012251

Shen, H. H., \& Squire, V. A. (1998). Wave damping in compact pancake ice fields due to interactions between pancakes. In (p. 325-341). Washington, DC, American Geophysical Union.

Smith, M., \& Thomson, J. (2019). Ocean surface turbulence in newly formed marginal ice zones. Journal of Geophysical Research: Oceans. doi: 10.1029/2018JC014405

Spreen, G., Kaleschke, L., \& Heygster, G. (2008). Sea ice remote sensing using amsre 89-ghz channels. Journal of Geophysical Research: Oceans, 113(C2). doi: 10 $.1029 / 2005 J C 003384$

Squire, V. A., Dugan, J. P., Wadhams, P., Rottier, P. J., \& Liu, A. K. (1995). Of ocean waves and sea ice. Annual Review of Fluid Mechanics, 27, 115-168. doi: 10.1146/annurev.fl.27.010195.000555

Sree, D. K., Law, A. W.-K., \& Shen, H. H. (2018). An experimental study on gravity waves through a floating viscoelastic cover. Cold Regions Science and Technology, 155, 289-299. doi: 10.1016/j.coldregions.2018.08.013

Tennekes, H., \& Lumley, J. L. (1972). A first course in turbulence. MIT press.

Thomson, J. (2012). Wave breaking dissipation observed with SWIFT drifters. Journal of Atmospheric and Oceanic Technology, 29(12), 1866-1882. doi: 10.1175/ JTECH-D-12-00018.1

Thomson, J. (2015). ONR Sea State DRI Cruise Report: R/V Sikuliaq, Fall 2015 (SKQ201512S) (Tech. Rep.). University of Washington.

Thomson, J., Ackley, S., Girard-Ardhuin, F., Ardhuin, F., Babanin, A., Boutin, G., ... Wadhams, P. (2018). Overview of the arctic sea state and boundary 
layer physics program. Journal of Geophysical Research: Oceans, 123(12), 8674-8687. doi: 10.1002/2018JC013766

Thomson, J., \& Rogers, W. E. (2014). Swell and sea in the emerging arctic ocean. Geophysical Research Letters, 41(9), 3136-3140. doi: 10.1002/2014GL059983

Wadhams, P., Squire, V. A., Goodman, D. J., Cowan, A. M., \& Moore, S. C.

(1988). The attenuation rates of ocean waves in the marginal ice zone. Journal of Geophysical Research: Oceans, 93(C6), 6799-6818. doi: 10.1029/JC093iC06p06799

Wang, R., \& Shen, H. H. (2010). Gravity waves propagating into an ice-covered ocean: A viscoelastic model. Journal of Geophysical Research: Oceans, 115(C6). doi: 10.1029/2009JC005591

Zippel, S., \& Thomson, J. (2016). Air-sea interactions in the marginal ice zone. Elementa Science of the Anthropocene, 4, 1-12. doi: 10.12952/journal.elementa .000095 


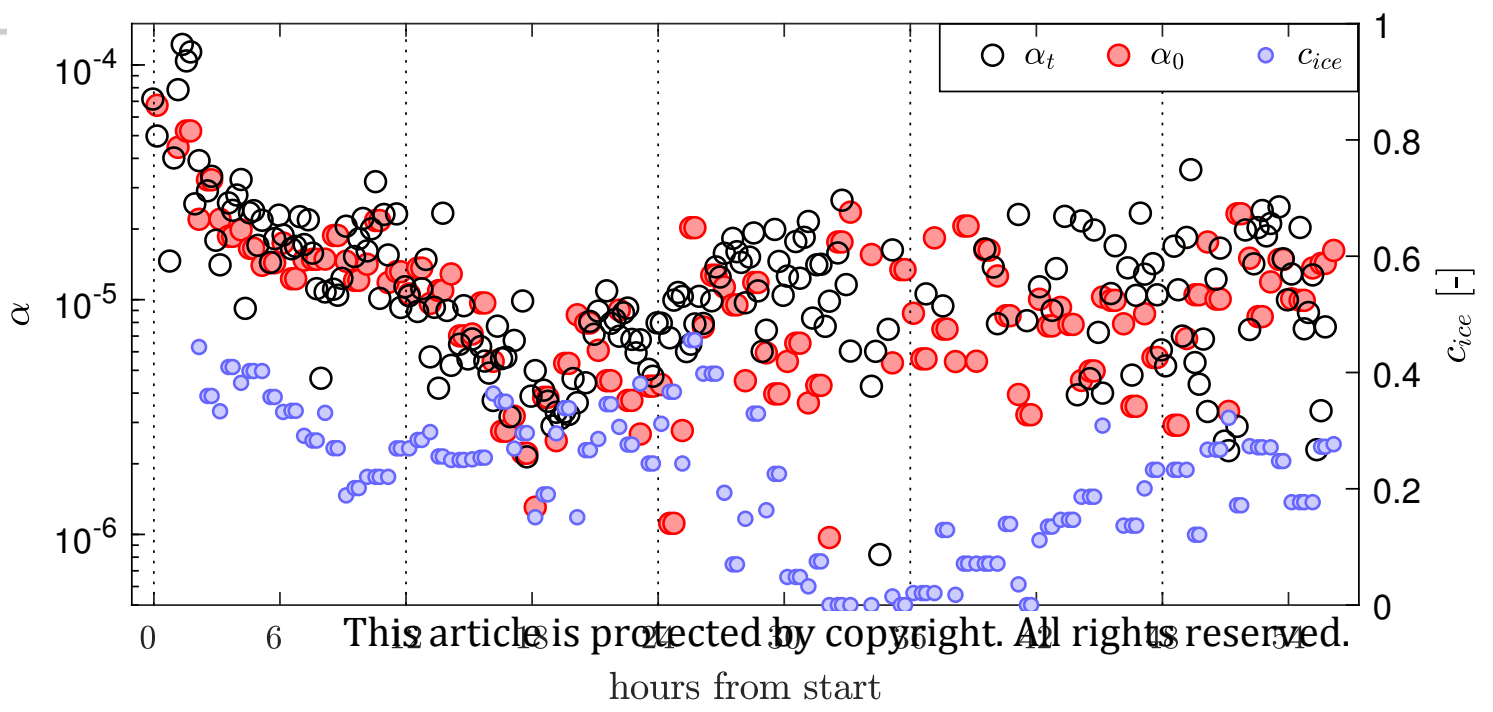


Figure 2.
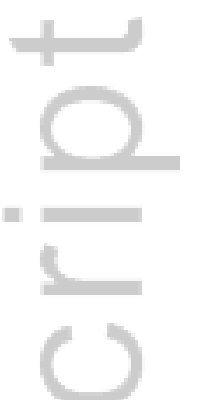

$\infty$
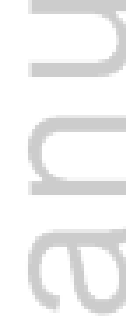

This article is protected by copyright. All rights reserved. 


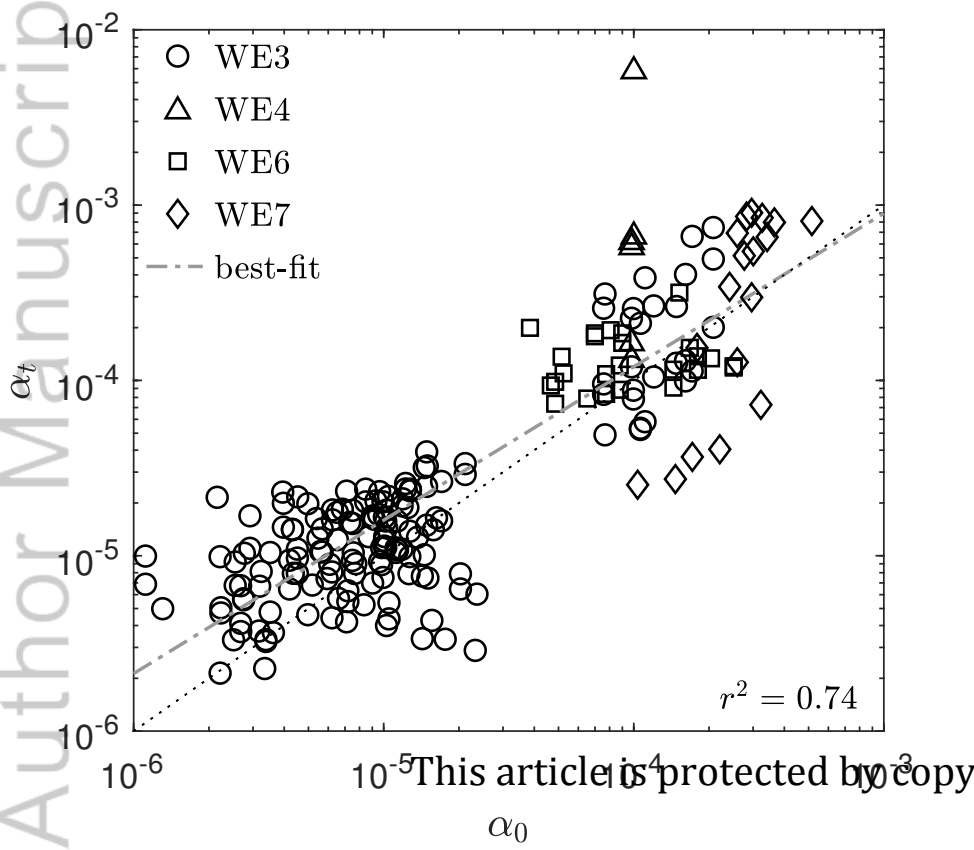




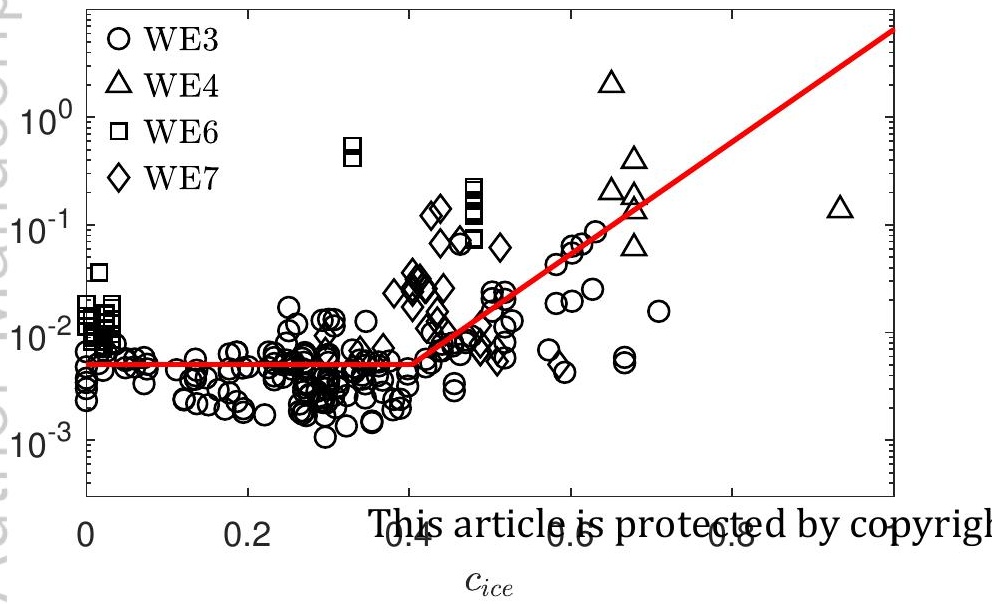




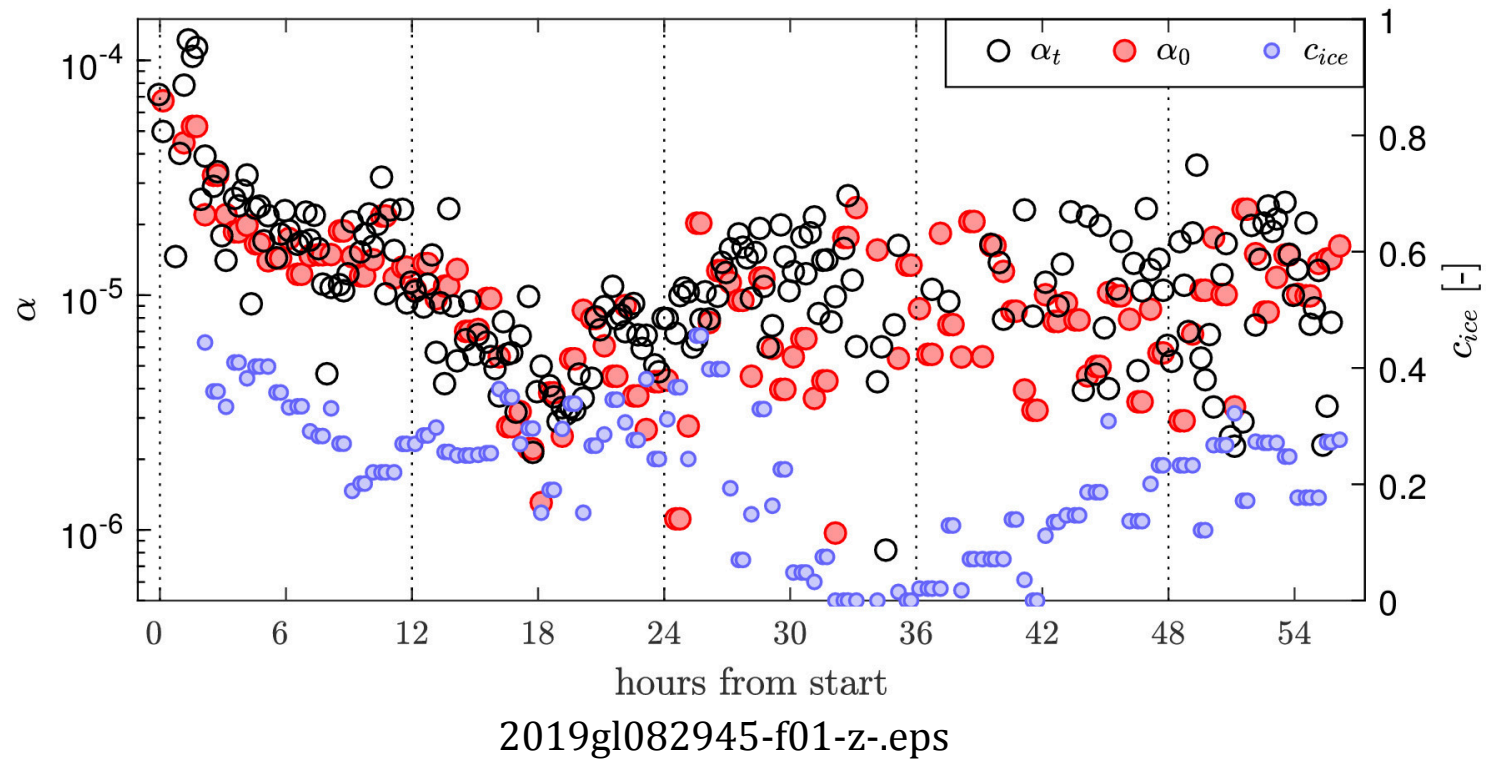

This article is protected by copyright. All rights reserved. 


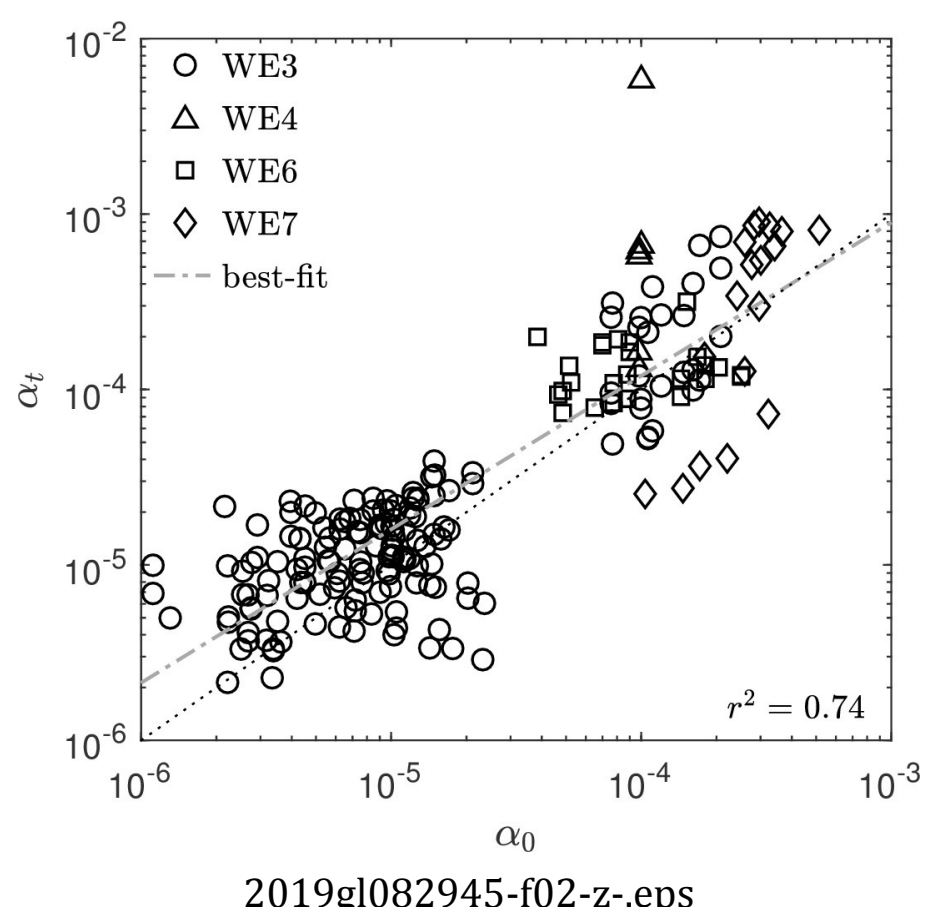

This article is protected by copyright. All rights reserved. 

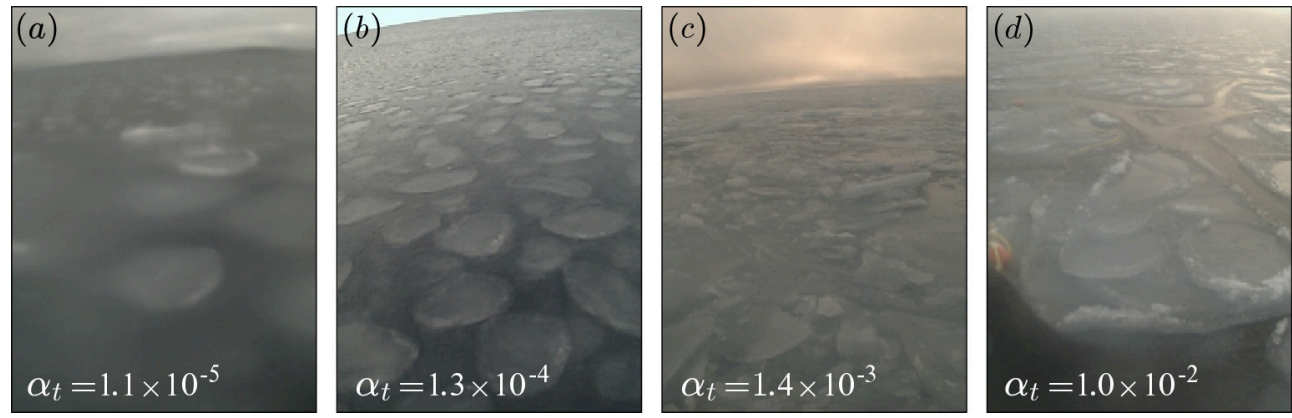

2019gl082945-f03-z-.eps

This article is protected by copyright. All rights reserved. 


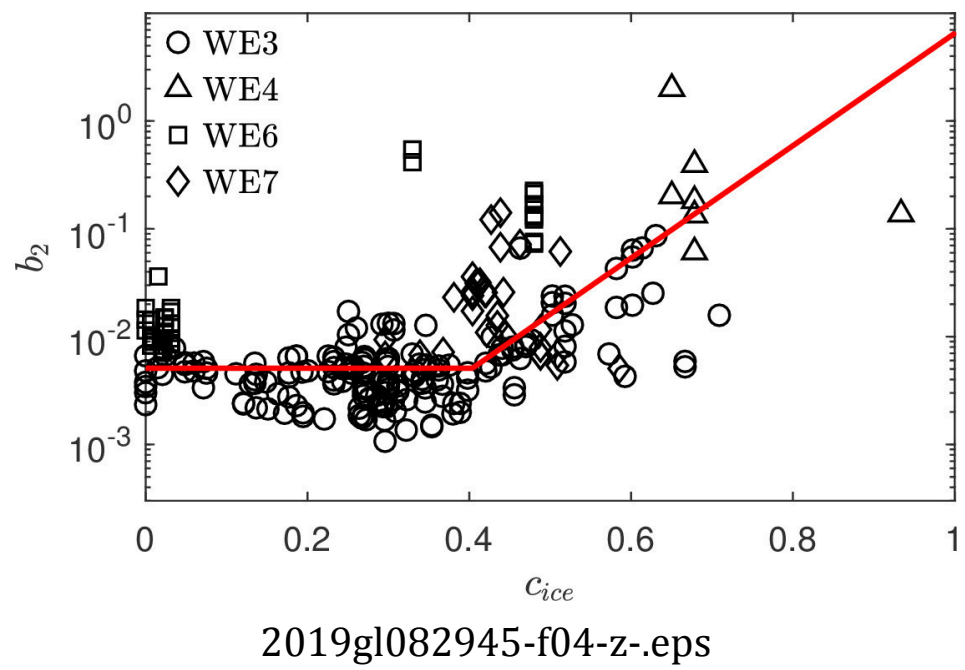

This article is protected by copyright. All rights reserved. 


\section{University Library}

\section{- M M I E E R VA A gateway to Melbourne's research publications}

Minerva Access is the Institutional Repository of The University of Melbourne

Author/s:

Voermans, JJ;Babanin, AV;Thomson, J;Smith, MM;Shen, HH

Title:

Wave Attenuation by Sea Ice Turbulence

Date:

2019-07-12

Citation:

Voermans, J. J., Babanin, A. V., Thomson, J., Smith, M. M. \& Shen, H. H. (2019). Wave Attenuation by Sea Ice Turbulence. Geophysical Research Letters, 46 (12), pp.6796-6803. https://doi.org/10.1029/2019GL082945.

Persistent Link:

http://hdl.handle.net/11343/285817 